


\title{
Inductively Coupled Small Self Resonant Coil (SSRC) Reader Antennas for HF RFID Applications
}

\author{
Soheyl Soodmand ${ }^{1}$ and Tim W.C. Brown ${ }^{2, *}$ \\ ${ }^{1}$ School of Electrical and Electronic Engineering, Newcastle University \\ ${ }^{2}$ Institute for Communication Systems (ICS), University of Surrey
}

\begin{abstract}
A new model of inductively coupled high frequency radio frequency identification (HF RFID) reader antennas is presented in this paper based on the idea of using the self resonance frequency (SRF) of a small multi turn coil. The introduced multi turn small self resonant coil (MT SSRC) antenna is mathematically analyzed in terms of SRF, number of turns, dimensions and dielectric characteristics of the insulation, where present. Based on the analysis, a compact planar version of MT SSRC antennas having two turns, the two turn planar SSRC (TTP SSRC), is investigated and the dependency of the SRF to the antenna dimension is observed. A TTP SSRC antenna operating at $13.56 \mathrm{MHz}$ is fabricated and is compared with an old model of HF RFID antennas; an optimized $Q$ factor and a more uniform near field pattern is obtained for the new antenna. The benefits of the obtained optimized $Q$ factor and uniform near pattern is explained for smart shelf application. Also, a number of TTP SSRC antennas operating at a distinct frequency, $13.56 \mathrm{MHz}$ here, are fabricated on different substrates and it is shown that the $Q$ factor and dimension of the TTP SSRC antenna could be controlled and adjusted based on the dielectric characteristics of the substrate. The new antenna prototype has a beneficial application to smart shelf applications in HF RFID.
\end{abstract}

\section{Introduction}

\subsection{Previous works}

In recent years automatic contact-less identification systems, known as radio frequency identification (RFID), have become very popular in purchasing, industry, tracking, transportation, sensing and many other applications. An RFID system is always made up of two components: Tag (transponder) and reader (interrogator). The tag is located on the object to be identified and contains electronically stored information, which can be read from up to several meters away by the reader. The first kind of RFID systems, which are out of the scope of this research, are long-range or far field systems having identification ranges significantly above $1 \mathrm{~m}$ (1-15m). Such systems operate using electromagnetic waves in the UHF and microwave range in the far field region of the reader antenna to identify the tagged objects. The second kind of the RFID systems, which use frequencies below $30 \mathrm{MHz}$ in the high frequency (HF) to operate, are named inductively coupled or HF RFID systems. The inductively coupled systems, which are in the scope of this research, have identification ranges of up to $1 \mathrm{~m}$. In these systems, an electrically small coil as a reader antenna uses its near magnetic field (H-field) to detect tagged items located in the identification range based upon magnetic coupling between the reader and the tag antenna coils [1] 


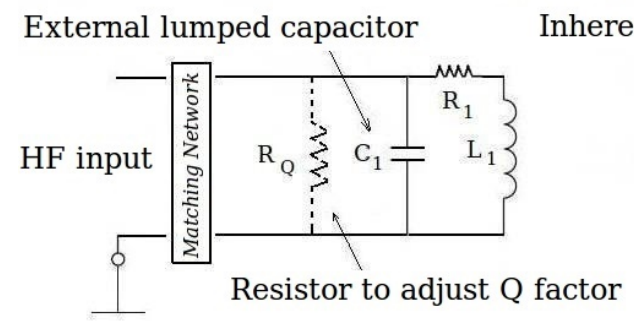

(a)

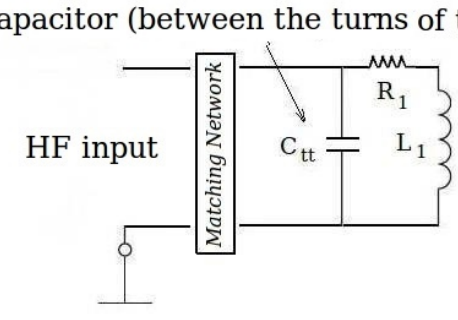

(b)

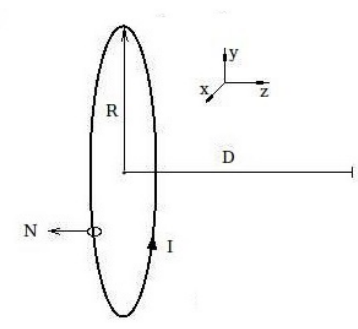

(c)

Fig. 1.

a Circuit diagram of the reader antenna of the inductive coupled system as an inductor coil operating below its self resonance frequency

b Circuit diagram of the reader antenna of the inductive coupled system as an inductor coil operating at its self resonance frequency.

c Reader loop antenna.

In the all of the previous inductively coupled/HF RFID systems the reader coil/loop antenna has been modeled as an inductor $L_{1}$ as shown in Fig.1(a) [1-7]. The series resistor, $R_{1}$, corresponds to the ohmic losses of the wire resistance of the coil which are insignificant for electrically small coils. By parallel connection of a lumped capacitor $C_{1}$ to the inductor $L_{1}$, a resonant circuit is created and the resonance frequency/operating frequency of the reader circuit is calculated using the Thomson equation as:

$$
f_{\mathrm{TX}}=\frac{1}{2 \pi \sqrt{L_{1} C_{1}}}
$$

The performance of an HF RFID antenna is related to its quality factor ( $Q$ factor). In general, the higher the $Q$ factor, the higher the power output for a particular sized antenna but too high a $Q$ factor may conflict with the band-pass characteristics of the reader and the increased ringing could create problems in the protocol bit timing. For these reasons the $Q$ factor of the HF RFID antenna is normally decreased to a proper value around 15 30 range, depending upon the required bandwidth and thus upon the modulation procedure used $[1,3,7]$, by adding a resistor, $R_{Q}$, parallel with the inductor as shown in Fig.1(a). The calculations of $R_{Q}$ are presented in [7] and an optimized system design based on numerical methods is presented in [3] for the inductively coupled RFID systems with high power demand. Also, in order to match the reader antenna to the fed source, which is normally $50 \Omega$, an impedance matching circuit at the input of the circuit in Fig.1(a) should be designed [1-3], [5-7].

As a reader antenna, a circular shape coil/loop shown in Fig.1(c), is the best choice to excite a magnetic alternating field at an inductively coupled system because the contributions of the current carrying parts of the circular loop arrive in phase at the tag location, resulting in constructive interference. $R, N$ and $D$ represent radius of reader loop antenna, number of coil turns and the distance between tag/reader coils, respectively. From the size point of view, the coils/loops used in the inductively coupled RFID systems are non-standard antennas in which their total wire length is small compared to the wavelength of the frequency of operation, $f_{\mathrm{TX}}$, (Total wire length $<0.1 \lambda$ ) because if the total wire length becomes a considerable part of the wavelength, the coil can not be considered as a lumped element; standing waves will cause multiple resonances and the total field will be decreased [1-2]. Based on previous literature, [1-2] and [8-11], effects of increasing 
$N$ on magnetic field intensity, $Q$ factor and self resonance frequency (SRF) are reviewed briefly in Section II and it is shown that the a single loop (one turn coil) is the best choice to be used in the model of Fig.1(a). It should be mentioned here that the previous attempts $[2,3,7,11]$ to design multi turn HF RFID coils with more than one turn to use in model of Fig.1(a) are carried out considering the $f_{\mathrm{TX}}<\mathrm{SRF}$ condition. The reason for this condition is discussed in Section II part B.

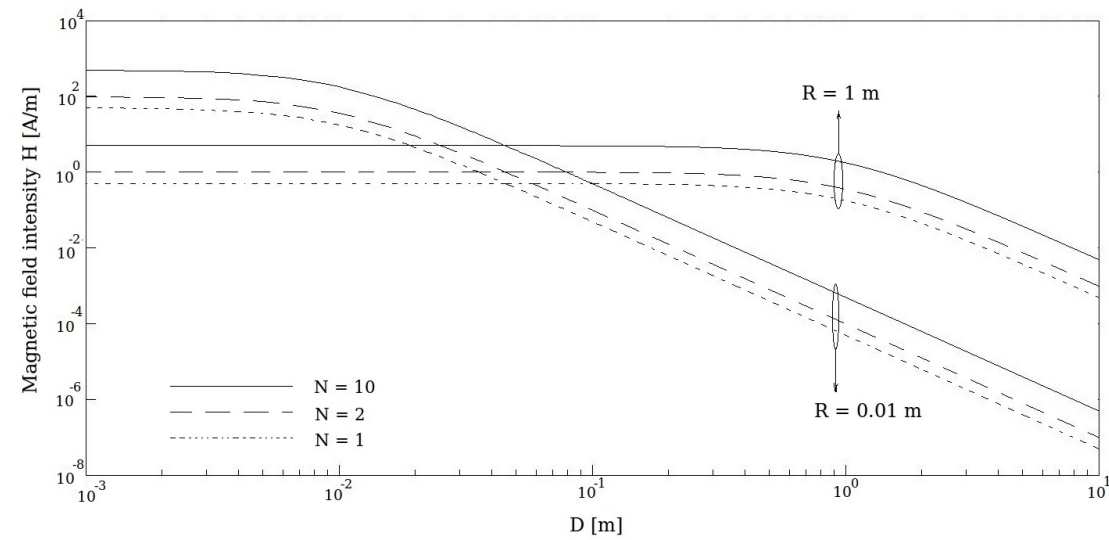

(a)

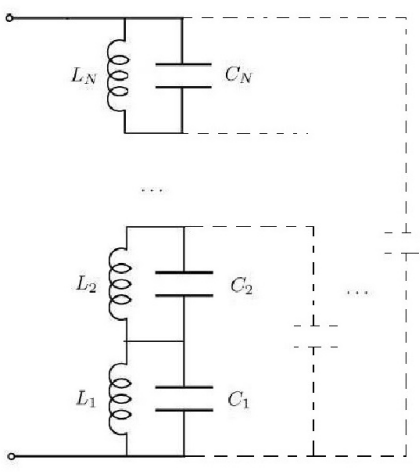

(b)

\section{Fig. 2.}

a $\left|H_{z}\right|$ as the distance in the $D$ direction is increased. $I=1 \mathrm{~A}$.

b Equivalent circuit of an inductor.

\subsection{This paper}

In this paper a new model is presented for the HF RFID reader antennas based on the idea of using the SRF of the small multi turn HF coil as the operating frequency of the reader, $f_{\mathrm{TX}}=\mathrm{SRF}$, as shown in Fig.1(b) i.e the inherent capacitance between the turns of the coil, instead of the external lumped capacitor of the model of Fig.1(a), is used to make resonance with the inductance of the coil. In Section III, multi turn small self resonant coil (MT SSRC) antennas operating at their SRF are analyzed mathematically at HF band considering insulated and non-insulated wires in their structure. To avoid an electrical short and also to get a compatible structure with the current HF RFID reader antennas by using the analysis done in Section III as an approximated procedure, a compact planar version of the insulated MT SSRC antennas having two turns, two turn planar SSRC (TTP SSRC), is proposed in Section IV and the dependency of the SRF to the antenna dimension is observed. A TTP SSRC with SRF=13.56 MHz following the model of Fig.1(b) and a single loop antenna following the model of Fig.1(a) having same dimension and operation frequency are fabricated and tested in Section V. It is shown that the TTP SSRC operating at $f_{\mathrm{TX}}=$ SRF has an optimized $Q$ factor nearer to the current specifications in the HF RFID standards (ISO/IEC 15693 and ISO/IEC 14443) and also a near field (H-field) pattern with more uniformity is obtained for the TTP SSRC antenna in comparison with the single loop that makes the TTP SSRC antenna a better choice to use in the intelligent HF RFID smart shelf application. Finally, in Section VI it is shown that the $Q$ factor and dimension of a TTP SSRC antenna operating at a distinct operating frequency, $13.56 \mathrm{MHz}$ for HF RFID, could be controlled and adjusted based on the characteristics of the dielectric substrate. Because the basic mathematical analysis of MT SSRC antennas are carried out for multi turn structures, it opens a gate to do more practical studies 
about MT SSRC antennas with more than two turns as a future work, while the measured and simulated results of the TTP SSRC antenna show good agreement with the analysis which are carried out in this paper.

\section{Review on multi-turn coils for HF RFID applications}

\subsection{Magnetic field and $Q$ factor}

Based on the Bio-Savart law of magneto-statics the current can be assumed to be near constant over the electrically small coils shown in Fig.1(c) so the amplitude of the magnetic field (H-field) in the direction perpendicular to the tag, at the distance $D$ is found as [12]:

$$
\left|\vec{H}_{z}\right|=\left|\frac{N I R^{2}}{2 \sqrt{\left(R^{2}+D^{2}\right)^{3}}}\right|
$$

It is shown that for the loop with radius $R$ the magnetic field of $\left|\overrightarrow{H_{z}}\right|$ at a distance $D=R / \sqrt{2}$ from the centre is maximal/optimal [1], [13-16]. However, $\left|\overrightarrow{H_{z}}\right|$ decreases rapidly as the distance in the $D$ direction is increased from $1 \mathrm{~mm}$ to $10 \mathrm{~m}$, as shown in Fig.2(a). The current ( $I=1 \mathrm{~A})$ is constant in each case; the coils differ in radius $R$ and number of turns $N$. Based on (2) and Fig.2(a), in the case the coil is fed with a current source, it is tempting to think that a high $N$ will result in a high magnetic field at the tag location when $R$ is constant, but it is shown in [2] that more turns are only advantageous for smaller $D(D<1 \mathrm{~m})$. The fact is that by increasing $N$, the total wire length of the loop which results can not be regarded small compared to the wavelength and for bigger $D$ the contributions of all parts of the loop at the tag location will not be in phase and therefore partial cancellation will occur over the loop. Also, when the coil is fed with a voltage source, calculations in [2] show that setting impedance of the coil in (2) for constant $R$ results in maximum value of $\left|\overrightarrow{H_{z}}\right|$ when $N=1$. Increasing $N$ will cause increased ohmic resistance as well, so the current in the loop wire would be even more confined than should be expected due to the skin effect. However, $\mathrm{H}$-field can be boosted by increasing the voltage/current of the fed source but the main problem for the HF RFID coil with more than one turn is that a high $Q$ factor will result $[1,2,3,7]$. It is shown in [2] that the $Q$ factor of a two turn coil reader antenna is too high in comparison with single turn coil as even at very small distances no communication can take place without using resistor $R_{Q}$.

\subsection{Self resonance frequency (SRF)}

When the perimeter of a conductor loop is about half a wavelength, the loop will resonate. If the loop has multiple turns, resonance occurs at a lower frequency due to the parallel resonance of the inductance of the loop and the parasitic capacitance (due to the electric field between the turns of wire which are at slightly different potentials). When designing a coil with $N>1$, to use in the model of Fig.1(a), the SRF of the coil should be checked to ensure it is higher than the reader operating frequency, i.e. $f_{\mathrm{TX}}<\mathrm{SRF}$, because above its SRF the coil starts to behave as a capacitor and the Thomson equation of (1) can not to be used. The SRF of HF coils has been reviewed mathematically here where the formulas are used in the sections to follow. The capacitance between two turns of the coil at HF band is calculated as [8]: 


$$
C_{t t}=\frac{2 \pi^{2} \varepsilon_{0} R}{\ln \left(\frac{d}{2 r}+\sqrt{\left(\frac{d}{2 r}\right)^{2}-1}\right)}
$$

where $R, r, d$ and $\varepsilon_{0}$ are loop radius, wire radius, distance between the centres of the two wires and permittivity of free space, respectively. If the wire has an insulation with a dielectric constant of $\varepsilon_{r} \neq 1$ the formula for coil at HF band is calculated as [9]:

$$
C_{t t}=\frac{2 \pi^{2} \varepsilon_{0} \varepsilon_{r} R}{\ln \left(1+\frac{t}{r}\right)}
$$

where the adjacent insulated turns are supposed to touch; without any air gap between them. The insulation thickness around the wire is shown by $t$. Substituting $\varepsilon_{r}=1$ into (4) does not result in (3) due to different approximations regarding [9] are used that led to (4).

The equivalent circuit of an inductor with multiple turns is shown in Fig.2(b). The equivalent capacitance is found as the series circuit of all turn-to-turn capacitances. As the capacitances between non-adjacent turns have a slight effect on the SRF, they are negligible at HF band and are not shown [2]. However, for a uniformly wound HF coil, $C_{1}=C_{2}=\ldots=C_{N}=C_{t t}$ and $L_{1}=L_{2}=\ldots=L_{N}=L_{1, N=1}=L_{1, N} / N . L_{1, N}$ and $C_{t t}$ indicate the inductance (in the presence of the other turns) and capacitance between two turns, respectively. $L_{1, N=1}$ is the inductance of a single turn in the absence of all other turns and is calculated as [10]:

$$
L_{1, N=1}=\mu_{0} R\left(\ln \left(\frac{8 R}{r}\right)-2\right)
$$

The SRF is calculated as [2]:

$$
\mathrm{SRF}=\frac{1}{2 \pi \sqrt{L_{1, N} C_{t t}}}=\frac{1}{2 \pi \sqrt{N L_{1, N=1} C_{t t}}}
$$

\section{Multi Turn Small Self Resonant Coil (MT SSRC) at HF Band}

Based on the discussion in Section II it is seen that the one turn coil $(N=1)$ is the best choice to use in the model of Fig.1(a), while all the previous attempts, [1,2,3,7,11], to design multi turn HF RFID coils using this model is done considering $f_{\mathrm{TX}}<\mathrm{SRF}$ condition and using the Thomson equation to define operation frequency. Here, a new model as shown in Fig.1(b) is presented for the HF RFID reader antenna based on the idea of transmitting at the SRF of the small HF coil with more than one turn, thus $f_{\mathrm{TX}}=\mathrm{SRF}$. Therefore the inherent capacitance between the turns of the multi turn coil is used instead of a lumped capacitor of model of Fig.1(a) to make a self resonance with the inductance of the coil. The coil is still considered to be small so the effect of increasing $N$ on magnetic field intensity is the same as Section II part A. Here, multi turn small self resonant coil (MT SSRC) antennas operating at their SRF are introduced and analyzed mathematically at the HF band considering insulated and non-insulated wires in their structure in terms of SRF, number of turns, dimension and dielectric constant (where insulated wire is used). Also it is shown mathematically that because of the possibility of an electrical short circuit, a noninsulated structure couldn't be used and an insulated structure should be used instead in order to get a practical structure for the MT SSRC antenna. 

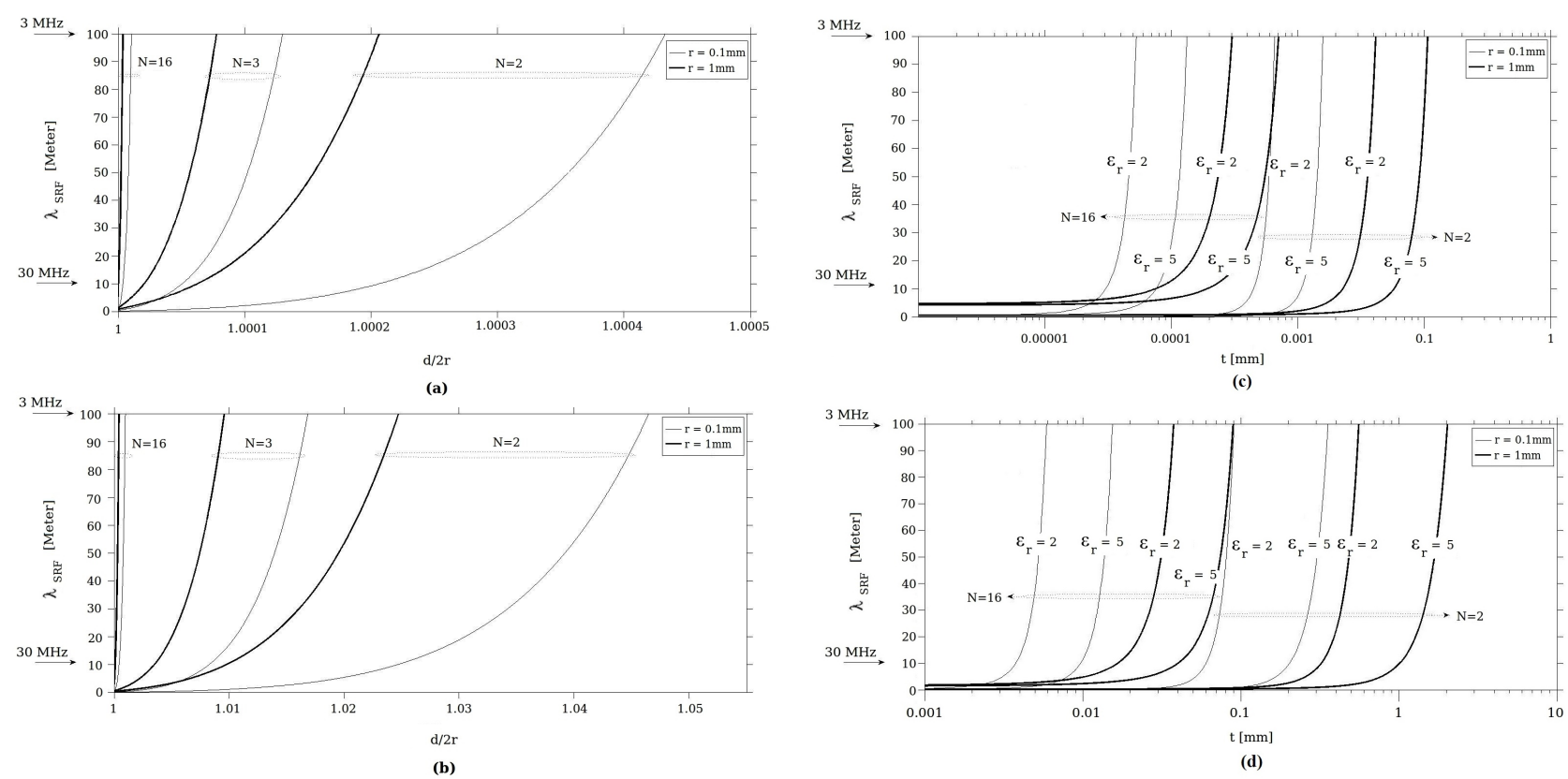

Fig. 3.

a $\lambda_{\mathrm{SRF}}$ vs $d / 2 r$ for non-insulated MT SSRC when $k=0.02$.

b $\lambda_{\mathrm{SRF}}$ vs $d / 2 r$ for non-insulated MT SSRC when $k=0.06$.

c $\lambda_{\mathrm{SRF}}$ vs $t$ for insulated MT SSRC when $k=0.02$.

$\mathrm{d} \lambda_{\mathrm{SRF}} \mathrm{vs} t$ for insulated MT SSRC when $k=0.06$.

\subsection{MT SSRC with non-insulated wires}

By substituting (3) and (5) into (6), knowing the fact that for transmission of the magnetic field at $\mathrm{SRF}, c=\mathrm{SRF} \cdot \lambda_{\mathrm{SRF}}=1 / \sqrt{\varepsilon_{0} \mu_{0}}$ where $c$ is speed of light in free space, the following equation is obtained for the wavelength of the SRF of the small HF coil having $N$ turns:

$$
\lambda_{\mathrm{SRF}}=\pi^{2} R \sqrt{8 N \frac{\ln \left(\frac{8 R}{r}\right)-2}{\ln \left(\frac{d}{2 r}+\sqrt{\left(\frac{d}{2 r}\right)^{2}-1}\right)}}
$$

The total wire length of a compressed coil with good approximation is taken equal to $2 \pi N R$ and in order to get the following equations simpler in terms of the electrical length of the coil the $k$ parameter is defined as $k=$ coil length $/ \lambda_{\mathrm{SRF}}=2 \pi N R / \lambda_{\mathrm{SRF}}$; it is obvious that for $k<0.1$ the antenna coil is small. However, replacing $R$ with $k \lambda_{\mathrm{SRF}} / 2 \pi N$ in (7) gives:

$$
\lambda_{\mathrm{SRF}}=\frac{\pi r N \exp (2)}{4 k}\left(\frac{d}{2 r}+\sqrt{\left(\frac{d}{2 r}\right)^{2}-1}\right)^{\frac{N}{\pi^{2} k^{2}}}
$$

Based on (8), $\lambda_{\mathrm{SRF}}$ of MT SSRC versus $d / 2 r$ is plotted for coils with $N=2,3,16$ and $r=0.1$, $1 \mathrm{~mm}$ characteristics having $k=0.02,0.06$ dimension factors at SRF $>3 \mathrm{MHz}$ or $\lambda_{\mathrm{SRF}}<100 \mathrm{~m}$ as shown in Fig.3(a) and Fig.3(b). It is seen that for $k=0.02$ and 0.06, which both allocate small dimensions for the MT SSRC, $d / 2 r$ is obtained around 1 with maximum amount of 1.05 at the HF band so $d=2 r$ would be a proper approximation for the dimension of the MT SSRC to have resonance at its SRF at the HF band considering the above specifications. The $d=2 r$ condition 
forces the adjacent turns being physically in touch in the structure of MT SSRC that causes an electrical short. Also, considering maximum value of $d=1.05(2 r)$ as distance between the turns leads to a very sensitive structure for the antenna even to very small mechanical stresses so MT SSRC antenna with non-insulated wires is not suitable for mass production. In Section III.B it is shown that MT SSRC antennas with insulated wires do not have such drawbacks and have good characteristics to be used for mass production where the printed version of this antenna introduced in Section IV make it more suitable case for mass production.

\subsection{MT SSRC with insulated wires}

If the coil is made of insulated wire with a relative permittivity of $\varepsilon_{r}$ and the thickness of $t$ for insulation then by replacing equations (4) and (5) into (6) the $\lambda_{\mathrm{SRF}}$ is obtained as:

$$
\lambda_{\mathrm{SRF}}=\pi^{2} R \sqrt{8 N \varepsilon_{r} \frac{\ln \left(\frac{8 R}{r}\right)-2}{\ln \left(1+\frac{t}{r}\right)}}
$$

As mentioned before in Section II part B, equation (9) is valid when the adjacent insulated turns are in contact without any air gap between them. Like Section III part A, $R$ is replaced in (9) with $k \lambda_{\mathrm{SRF}} / 2 \pi N$ so $\lambda_{\mathrm{SRF}}$ is obtained as:

$$
\lambda_{\mathrm{SRF}}=\frac{\pi r N \exp (2)}{4 k}\left(1+\frac{t}{r}\right)^{\frac{N}{2 \varepsilon_{r} \pi^{2} k^{2}}}
$$

Based on (10), $\lambda_{\mathrm{SRF}}$ of insulated MT SSRC versus insulation thickness of $t$ is plotted in logarithmic scale when $N$ is equal to 2 and 16; $r$ is fixed at 0.1 and $1 \mathrm{~mm}$ and $\varepsilon_{r}$ has values of 2 and 5 where $k$ is consequently equal to 0.02 and 0.06 at the HF band as shown in Fig.3(c) and Fig.3(d). In this case, there is no possibility of an electrical short circuit due to the adjacency of insulated turns. For example for the insulated MT SSRC having specifications of $N=2, k=0.06$ and $\varepsilon_{r}=2$ when $r$ is $0.1 \mathrm{~mm}$ or $1 \mathrm{~mm}$ the insulation thickness should be around $t=0.07 \mathrm{~mm}$ or $0.4 \mathrm{~mm}$, respectively, to achieve an SRF at $13.56 \mathrm{MHz}\left(\lambda_{\mathrm{SRF}}=22 \mathrm{~m}\right)$.

\section{Two turn planar SSRC (TTP SSRC) Antennas at HF band}

As seen in Section III part A, using the non-insulated wires in the structure of the MT SSRC causes an electrical short circuit so to avoid this and also to have a compatible structure with the current HF RFID reader antennas, a compact planar version of insulated MT SSRC antennas having two turns is proposed here based on the analysis in Section III part B as an approximated design procedure - considering the fact that as the antenna is electrically very small a planar approximation from wire structure wouldn't have a major effect as following simulations and measurements satisfy this. Based on the results shown in Fig.3(d), an insulated MT SSRC antenna with $k=0.06, N=2$ and dielectric constant of $\varepsilon_{r}=5$ for the insulation is considered as an example to be tried in planar structure. For these specifications from Fig.3(d) it is seen that when $r=0.1 \sim 1 \mathrm{~mm}$ the insulation thickness of $t$ should be at $0.2 \sim 2 \mathrm{~mm}$ range to get the SRF at the HF band $(3 \mathrm{MHz} \sim 30 \mathrm{MHz})$ for the MT SSRC antenna with $N=2$. FR4 substrate with a dielectric constant of $\varepsilon_{r}=4.4$ and thickness of $h=2 t=0.8 \mathrm{~mm}$, which are similar characteristics with what is mentioned above for the insulation, is selected for the two turn planar SSRC (TTP SSRC) having $k=0.06$ dimension to be mounted on. Because adjacent turns in contact without any air gap are considered for the insulated MT SSRC, as mentioned in Section III part B, the dielectric thickness of the substrate 


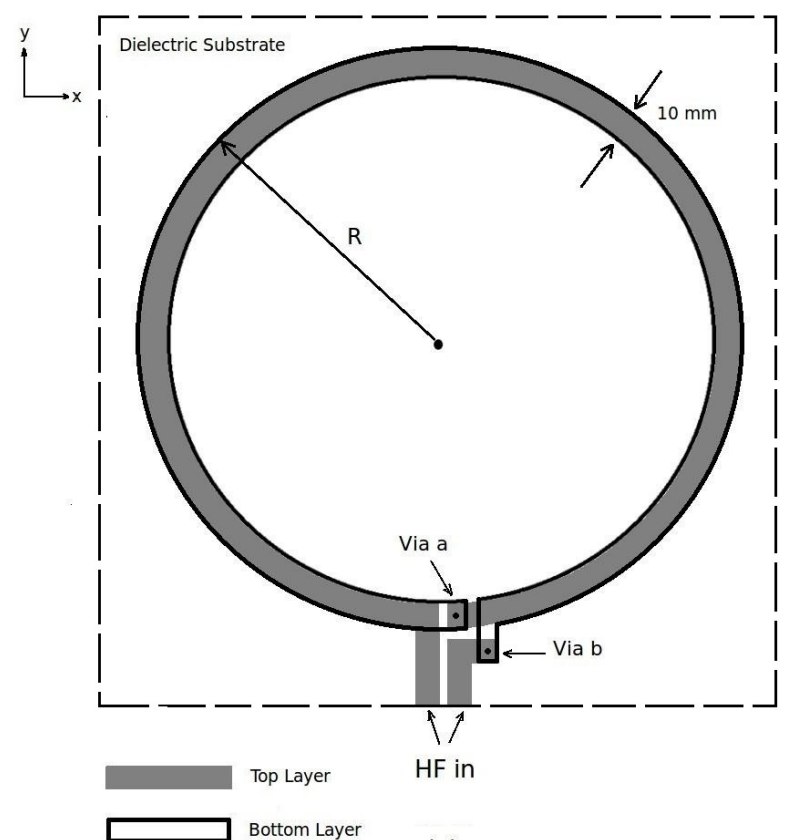

(a)

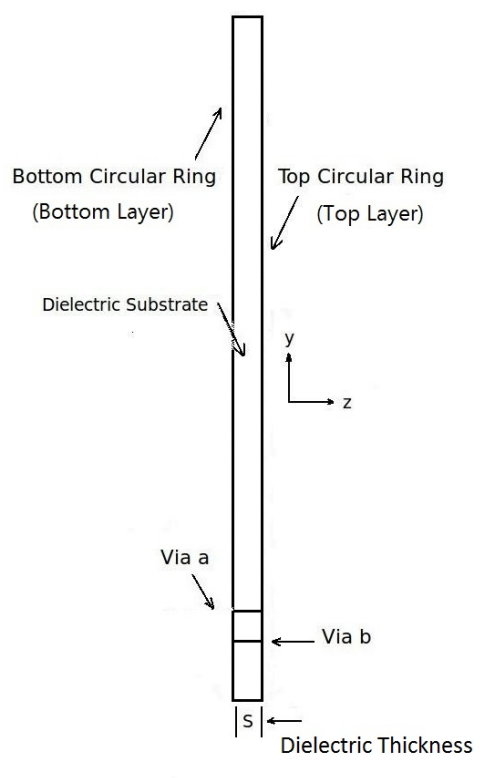

(b)

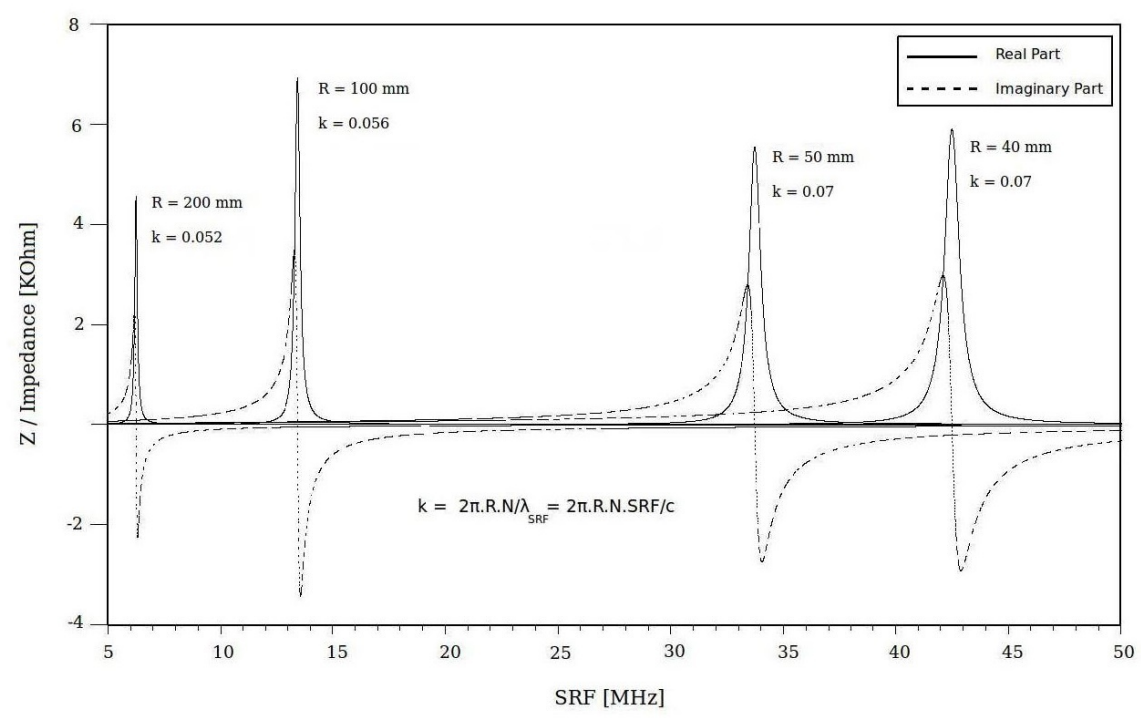

(c)

Fig. 4.

a Geometry of the TTP SSRC antenna, Top and bottom layers.

b Geometry of the TTP SSRC antenna, Side view.

c Impedance characteristics of the TTP SSRC antenna for different values of $R$.

is approximated with the twice of the insulation thickness i.e $h=2 t$. The TTP SSRC antenna structure is mounted as two circular copper strip loops having the same radius of $R$ and the same strip width of $10 \mathrm{~mm}$ on the top and bottom of the FR4 substrate, as shown in Fig.4(a) and Fig.4(b). In order to obtain a semi coil structure the two loops are connected together by via hole, $a$, in the 


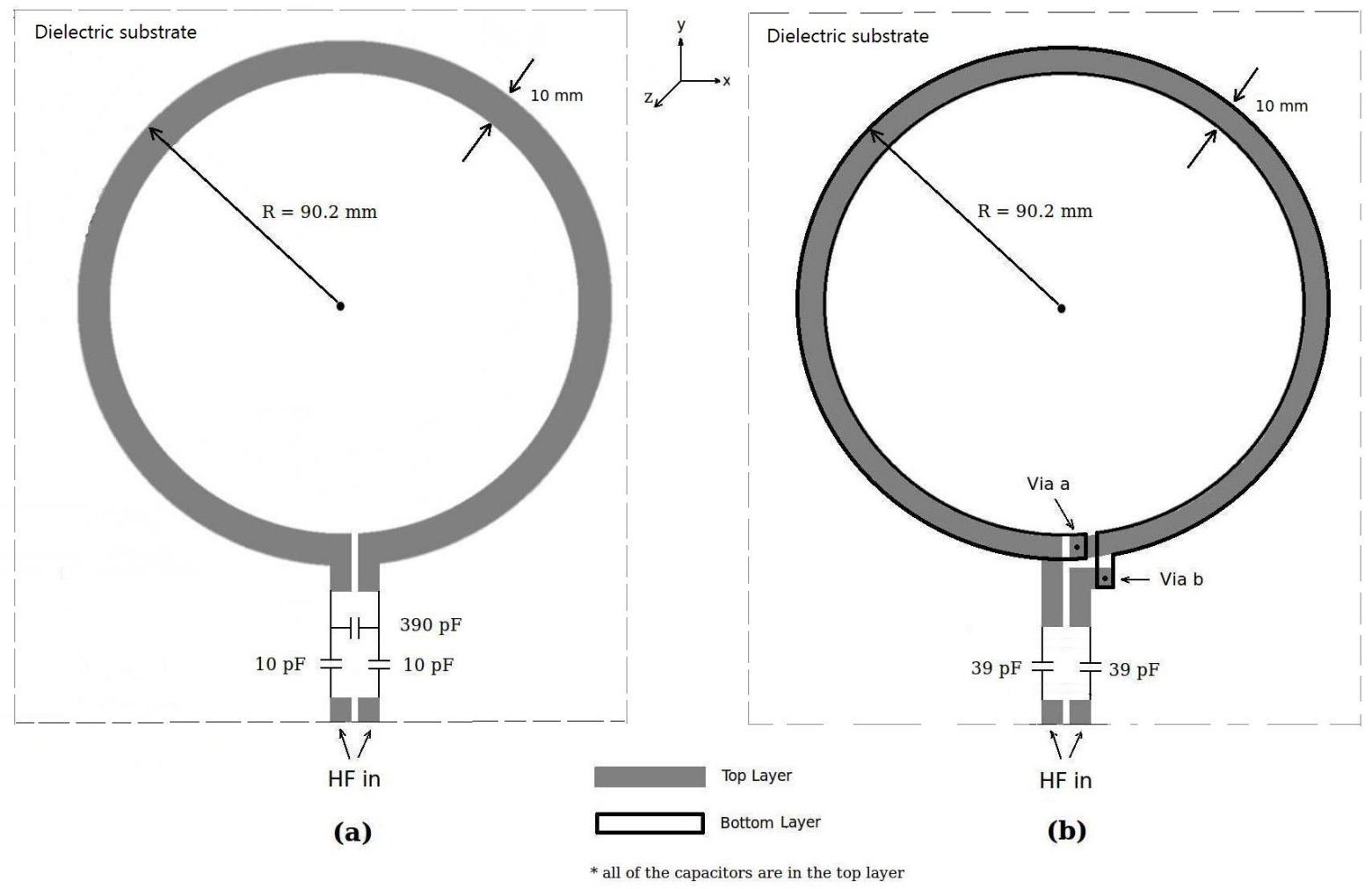

Fig. 5. Geometries of the (a) Planar single loop antenna (b) TTP SSRC antenna (both are designed to operate at $13.56 \mathrm{Mhz}$ and are matched to $50 \Omega$ input).

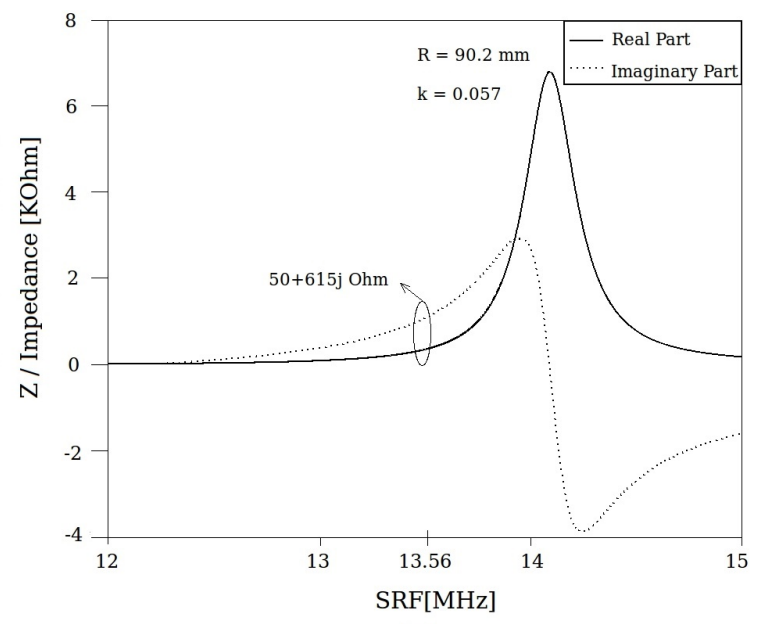

(a)

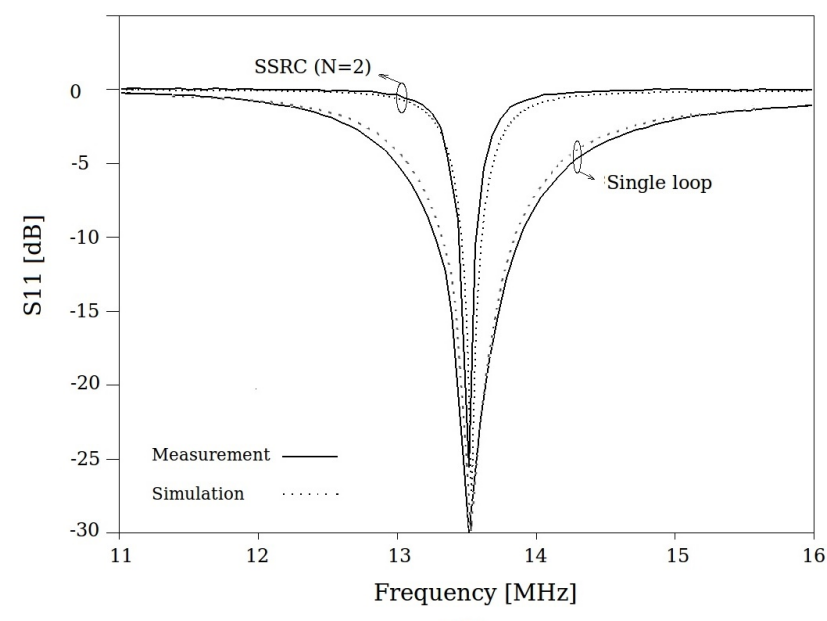

(b)

Fig. 6.

a Impedance characteristics of the TTP SSRC antenna, Fig.5(b), for $R=90.2 \mathrm{~mm}$.

$\mathrm{b}$ Simulated and measured return losses of the TTP SSRC antenna and single turn antenna both operating at $13.56 \mathrm{MHz}$, Fig.5. 
substrate, also via $b$ is used in the same way to connect the bottom loop to the antenna feed in the top layer. Impedance response of the antenna is simulated by HFSS software for different values of $R$ and is shown in Fig.4(c). It is seen that when $R$ decreases gradually from $200 \mathrm{~mm}$ to 40 $\mathrm{mm}$, SRF increases from $6 \mathrm{MHz}$ to $43 \mathrm{MHz}$, which shows the dependency of the SRF on the TTP SSRC antenna dimension. As shown in Fig.4(c), $k$ using $k=2 \pi N R / \lambda_{\mathrm{SRF}}=2 \pi N R \cdot \mathrm{SRF} / c$ expression is calculated around $k=0.06$ for all of the simulated antennas that follows the analyses in Section III part B. As seen in Fig.4(c) the real impedance peak value at the all of SRFs is about $6 \mathrm{k} \Omega$. Simulations, not shown here, depict that for $w=5 \mathrm{~mm}$ and $15 \mathrm{~mm}$ there is about $15 \%$ shift in the SRFs with the real impedance peak values of $8 \mathrm{k} \Omega$ and $5.5 \mathrm{k} \Omega$, respectively.

\section{TTP SSRC antenna operating at $13.56 \mathrm{MHz}$}

Following the above section, a TTP SSRC antenna having $k=0.06$ dimension is fabricated on FR4 substrate with $\varepsilon_{r}=4.4$ and $h=0.8 \mathrm{~mm}$ characteristics, Fig.5(b), to operate at $13.56 \mathrm{MHz}$, which is the one most frequently used for HF RFID systems [1]. Because real peak value of the impedance at the SRF is around $7 \mathrm{k} \Omega$, too far from $50 \Omega$, the matched antenna with a $P I$ or $T$ matching network will have a very high $Q$ factor to operate exactly at SRF [17]. In order to solve this problem the operating frequency of $13.56 \mathrm{MHz}$ is considered at the beginning of the real impedance curve when SRF $=14.1 \mathrm{MHz}(R=90.2 \mathrm{~mm})$ as shown in Fig.6(a). ${ }^{1}$ The impedance of the TTP SSRC antenna at $13.56 \mathrm{MHz}$ is around $50+615 \mathrm{j} \Omega$ when $\mathrm{SRF}=14.1 \mathrm{MHz}$ and the inductive part of $615 \mathrm{j}$ $\Omega$ is eliminated by just adding two serious capacitors of $39 \mathrm{pF}$ to match the antenna to $50 \Omega$ line, as shown in Fig.5(b).

Also based on the old model shown in Fig.1(a), a planar single loop HF RFID antenna having the same radius of $R=90.2 \mathrm{~mm}$ with the TTP SSRC having $k=0.06$ dimension is designed and fabricated to operate at $13.56 \mathrm{MHz}$. Following the Thomson formula by adding a $390 \mathrm{pF}$ capacitor to the input of the single loop a resonance LC circuit is created to operate at $13.56 \mathrm{MHz}$ and also a $50 \Omega$ matching circuit is designed as described in $[5,6]$ and illustrated in Fig.5(a). From the measurement/simulation results of return loss parameter shown in Fig.6(b), the $Q$ factor of the both antennas are calculated using the $f_{\text {centre }} /\left(f_{\text {high }-3 \mathrm{~dB}}-f_{\text {low }-3 \mathrm{~dB}}\right)$ expression and are obtained as 25 and 8 for TTP SSRC antenna and single loop antenna, respectively. It is seen that the $Q$ factor of the TTP SSRC antenna following the new model of Fig.1(b), $f_{\mathrm{TX}}=\mathrm{SRF}$, is nearer to the specifications of the standard $13.56 \mathrm{MHz}$ systems at $15 \sim 30$ range ([1,3,7], ISO 14443, ISO 15693).

The measured relative magnetic field intensity for the single loop and the TTP SSRC antenna at different distances in front of three points on the antenna, A,B and C, up to 1 metre is shown in Fig.7(a)and Fig.7(b) (With more accuracy for TTP SSRC antenna at $0 \sim 10 \mathrm{~cm}$ range in Fig.7(c)). From Fig.7(a)and Fig.7(b) it is seen that the H-field is more uniform in front of the TTP SSRC antenna than the single loop planar antenna so thinner tagged objects could be identified as well and the distance from the loop could be predicted with more accuracy in front of the TTP SSRC antenna. This is crucial for the smart shelf application such that it can determine the position of the item of the shelf. Also, in front of a uniform near field pattern there would be more degrees of freedom to change the position of the tag on the object's surface. Also, the measured relative magnetic field intensity of TTP SSRC antenna is about $5 \mathrm{~dB}$ stronger than single loop antenna in front of the three points at the constant distance levels that follows the discussion of Section II part

\footnotetext{
${ }^{1}$ It is seen that $\mathrm{SRF}(14.1 \mathrm{MHz})$ / operation frequency $(13.56 \mathrm{MHz})=1.04$ which is near to 1 so we still could say with a good approximation that $\mathrm{SRF}=$ operation frequency in our case which is without parallel resonance capacitor.
} 
A.

In order to measure relative magnetic intensity when adjacent tags are placed in front of TTP SSRC reader antenna, a set up as shown in Fig.8(a) is considered. Tag 1 is connected to spectrum analyser and placed at distance $M$ in front of the TTP SSRC antenna and tag 2 is placed at distance $P$ from tag 1 , toward reader antenna. In four individual measurements, tag 1 is fixed at $M=10$, $15,20,30 \mathrm{~cm}$ distances and the position of tag 2 is changed at $0 \sim 10 \mathrm{~cm}$ range. The measurement results obtained by spectrum analyser in front of the TTP SSRC reader antenna are shown in Fig.8(a) with about $1 \mathrm{~dB}$ difference for points $\mathrm{A}$ and C. It is seen that the adjacent tag 2 affects the measured magnetic field about $1 \mathrm{~dB}$ when is placed in less than $1.5 \mathrm{~cm}$ distance from the tag 1 and it has no effect on the measured magnetic field far away from this distance.

\section{Adjusting $Q$ factor and dimension of TTP SSRC antenna by dielectric characteristics of the substrate}

It is shown in [1] that the $Q$ factor of the single loop is dependent on the loop radius $R$, which increases the $Q$ factor. In this section dependency of the $Q$ factor of the TTP SSRC antenna to the dielectric characteristics of the substrate is investigated and it is shown that the $Q$ factor of the TTP SSRC antenna could be controlled based on these characteristics. Seven TTP SSRC antennas all operating at $13.56 \mathrm{MHz}$ are designed and fabricated on substrates having different dielectric characteristics. Fig.8(b) shows one of the measurement setups and the measured results are summarized in Table I. It is seen that the dominant factor to control the $Q$ factor is the loss tangent or dissipation factor of the dielectric as by increasing loss tangent the $Q$ factor is decreased, like the same effect for the lumped capacitors [18] (more details on the relationship between $\mathrm{Q}$ factor and energy is given in [19-22]). Also, the thickness of the dielectric affects the $Q$ factor, for example compare the $Q$ factor of the antennas fabricated on RT / Duroid 5880 and RT / Duroid 5870 having about the same loss tangent. The dielectric constant also affects the TTP SSRC antenna dimension (dielectric length) and as is seen in Table I more dielectric constant results in a smaller dimension or $R$.It is seen that loss tangent, dielectric thickness and dielectric constant all could be considered to control the $Q$ factor and dimension of the TTP SSRC antenna operating in a defined frequency. This makes the introduced antenna a suitable echoic for HF RFID smart shelf applications where different dimensions are needed based on the identifiable objects size.

Table 1 Measured $Q$ factor and dimension of seven TTP SSRC antennas, all having the same operating frequency of $13.56 \mathrm{MHz}$, fabricated on substrates with different loss tangent, thickness and dielectric constant parameters.

\begin{tabular}{|c|c|c|c|c|c|c|}
\hline $\begin{array}{c}\text { loss } \\
\text { tangent } \\
(\tan \delta)\end{array}$ & $\begin{array}{c}\mathrm{h} \\
(\mathrm{mm})\end{array}$ & $\mathrm{Q}$ & $\begin{array}{c}-10 \mathrm{~dB} \mathrm{BW} \\
(\mathrm{KHz})\end{array}$ & $\varepsilon_{r}$ & $\begin{array}{c}\mathrm{R} \\
(\mathrm{mm})\end{array}$ & Substrate \\
\hline 0.0004 & 0.25 & 670 & 6.5 & 2.2 & 82 & $\mathrm{RT} /$ Duroid 5880 \\
\hline 0.0005 & 1.6 & 275 & 17 & 2.3 & 149 & RT/ Duroid 5870 \\
\hline 0.0014 & 0.5 & 265 & 20 & 3 & 93.3 & Arlon /AD 300A \\
\hline 0.002 & 0.64 & 225 & 24 & 10.2 & 64 & RT / Duroid 6010 \\
\hline 0.0175 & 0.8 & 25 & 120 & 4.55 & 92 & FR4 \\
\hline 0.0175 & 1.6 & 20 & 130 & 4.55 & 112 & FR4 \\
\hline 0.045 & 1.6 & 16 & 260 & 4.2 & 110 & Econoboard CEM/1 \\
\hline
\end{tabular}




\section{Conclusion}

In the presented new model of inductively coupled reader antennas, SRF was used as operating frequency of HF RFID system and the introduced small multi turn SSRC (MT SSRC) antenna were analyzed. The dependency of the SRF to the antenna dimension was observed for compact planar version of MT SSRC antennas with two turns i.e. two turn planar SSRC (TTP SSRC) antennas. Several TTP SSRC antennas operating at $13.56 \mathrm{MHz}$ frequency were fabricated on different substrates and it was shown that the $Q$ factor and dimension of the TTP SSRC antenna operating at a distinct frequency, $13.56 \mathrm{MHz}$ here, could be controlled and adjusted based on the dielectric characteristics of the substrate. Also, a comparison was made between the TTP SSRC antenna presented in this paper and the old model of HF RFID antenna and it was shown that optimized $Q$ factor - for ISO/IEC 15693 and ISO/IEC 14443 standards - and more uniform near field pattern could be obtained for the presented antenna. All the measured and simulated results for TTP SSRC antenna show good agreement with the mathematical analysis.

\section{References}

[1] Finkenzeller, F.: 'RFID Handbook Fundamentals and Applications in Contactless Smart Cards, Radio Frequency Identification and Near - Field Communication' (New York: Wiley, 3rd edn. 2010)

[2] Aerts, W., De Mulder, E., Preneel, B., Vandenbosch G. A .E., Verbauwhede, I. : 'Dependence of RFID Reader Antenna Design on Read Out Distance', IEEE Trans. Antennas Propag.,2008, 56, (12), pp. 3829-3837

[3] Reinhold, C., Scholz, P., John, W., Hilleringman, U. : 'Efficient antenna design of inductive coupled RFID-systems with high power demand', Journal of Communications, 2007, 2, (6), pp. 14-23

[4] Scholz, P., Ackermann, W., Weil,T., Reinhold, C.: 'Antenna modeling for inductive RFID applications using the partial element equivalent circuit method',IEEE Trans. Magn., 2010, 46, (8), pp. 2967-2970

[5] Qing, X., Chen,Z. N.: 'Characteristics of a metal-backed loop antenna and its application to a high-frequency RFID smart shelf', IEEE Antennas Propag. Mag, 2009, 51, (2), pp. 26-28

[6] Qing, X., Chen, Z. N.: 'Proximity effects of metallic environments on high frequency RFID reader antenna: study and applications', IEEE Trans. Antennas Propag., 2007, 55, (11), pp. 3105-3111

[7] 'HF Antenna Design Notes-Technical application report-radio frequency identification systems' (Texas Instruments, 2003), pp. 9-23

[8] Magnusson,P.C., Alexander, G.C., Tripathi, V.K., and Weisshaar,A.: 'Transmission Lines and Wave Propagation'(CRC Press, 4th edn. 2001)

[9] Gabriele Grandi,A. M., Kazimierczuk,M. K., Reggiani,U.: 'Stray capacitances of single-layer solenoid air-core inductors', IEEE Trans. Ind. Appl., 1999, 35, (5), pp. 1162-1168

[10] Gover, F.W.: 'Inductance Calculations: Working Formulas and Tables'(Dover Publications Inc., 2009) 
[11] Tak,Y., Park,J., Nam,S.: 'The optimum operating frequency for near-field coupled small antennas', IEEE Trans. Antennas Propag., 2011, 59, (3), pp. 1027-1031

[12] Choudhury, M. H.: 'Electromagnetism' (Ellis Horwood,1989)

IET.,'Report Title' (Publisher, 2013), pp. 1-5

[13] AN678., 'RFID coil design'( Microchip Tech. Inc, 1998), pp. 1-20

[14] Hsu, H.M., Huang, W.Z., Lee, G.W., Chen, C.Y: 'Coil distance effect in magnetic resonance power transfer system', 2016 IEEE Int. Symp. on Radio-Frequency Integration Technology (RFIT), Taipei, Taiwan, August 2016, pp. 1-3

[15] Laskoski, G. T., Pichorim, S. F., Abatti, P. J.:'Distance Measurement With Inductive Coils', IEEE Sensors Journal, 2012, 12, (6), pp. 2237-2242

[16] Mastri, F., Costanzo, A., Mongiardo, M.: 'Coupling-Independent Wireless Power Transfer', IEEE Microwave and Wireless Components Letters, 2016, 26, (3), pp. 222-224

[17] Orfanidis, S.J.: 'Electromagnetic Waves and Antennas' (Rutgers University,2010)

[18] [Online]. Available: http://www.radio-electronics.com/info/formulae/ capacitance/esr-dfloss-tangent-q-tutorial-basics.php, accessed 5 August 2016

[19] Capek, M., Jelinek, L., Hazdra, P.: 'On the functional relation between quality factor and fractional bandwidth', IEEE Trans. Antennas Propag., 2015, 63, (6), pp. 2787-2790

[20] Yaghjian, A.D, Best,S.R.: 'Impedance, bandwidth, and Q of antennas', IEEE Trans. Antennas Propag.,2005,53, (4), pp. 1298-1324

[21] Li, N., Cao, M. , Liu, K. , He, C. , Wu, B. : 'A boundary detecting method for post-tensioned pre-stressed ducts based on Q-factor analysis', AEU - International Journal of Electronics and Communications ,2016,248, pp. 8893

[22] Xi, X., Wu, X., Zhang, Y., Zhou, X., Wu, X. , Wu, X. : 'A study on Q factor of the trimmed resonator for vibratory cupped gyroscopes', AEU - International Journal of Electronics and Communications ,2014,218, pp. 2332 


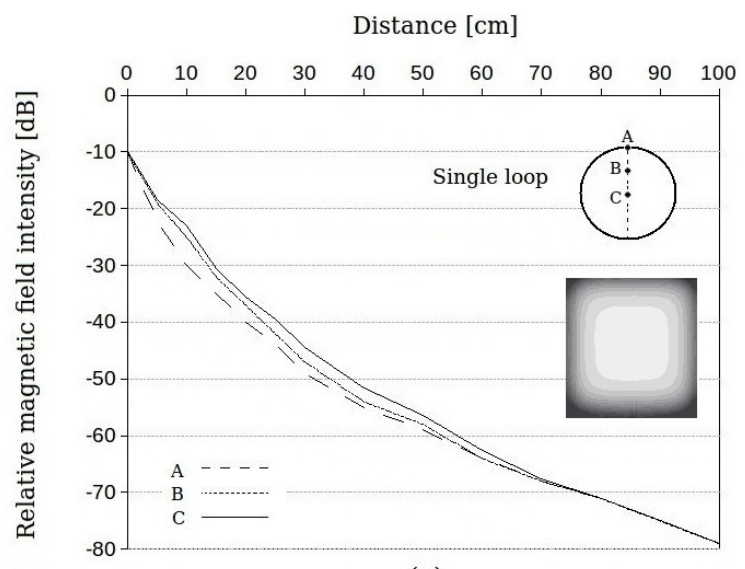

(a)

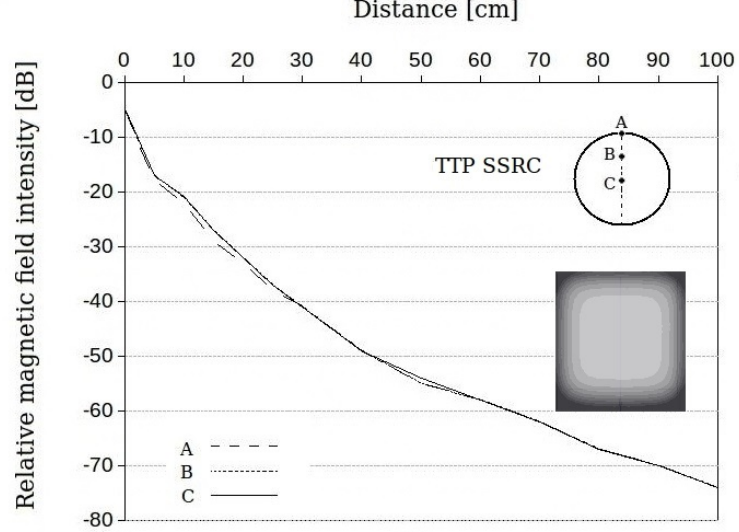

(b)

Distance $[\mathrm{cm}]$

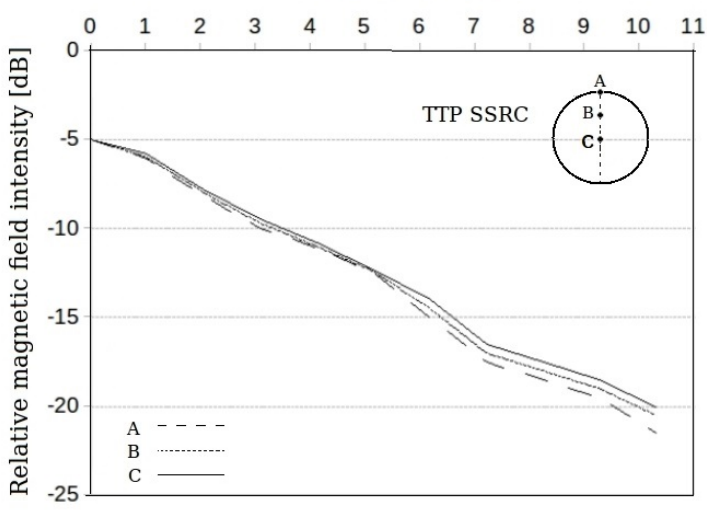

(c)

Fig. 7.

a The measured relative magnetic field intensity for the single loop antenna at different distances in front of three points of $\mathrm{A}, \mathrm{B}$ and $\mathrm{C}$, up to 1 metre distance. Also, simulated Magnetic filed distribution plot is shown at $50 \mathrm{~cm}$ distance.

$\mathrm{b}$ The measured relative magnetic field intensity for the TTP SSRC antenna at different distances in front of three points of $\mathrm{A}, \mathrm{B}$ and $\mathrm{C}$, up to 1 metre distance. Also, simulated Magnetic filed distribution plot is shown at $50 \mathrm{~cm}$ distance.

c The measured relative magnetic field intensity for TTP SSRC antenna at different distances in front of three points of $\mathrm{A}, \mathrm{B}$ and $\mathrm{C}$, up to $10 \mathrm{~cm}$ distance. 


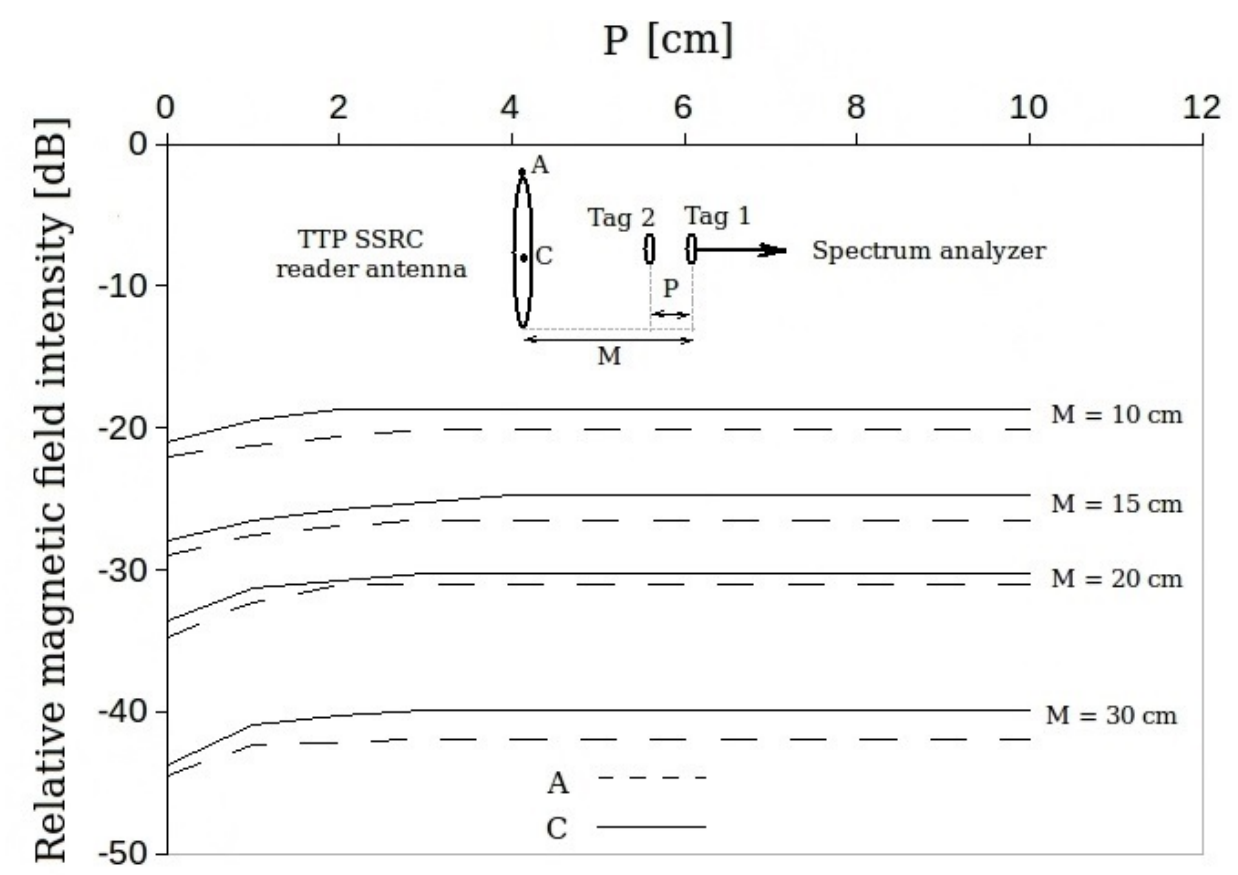

(a)

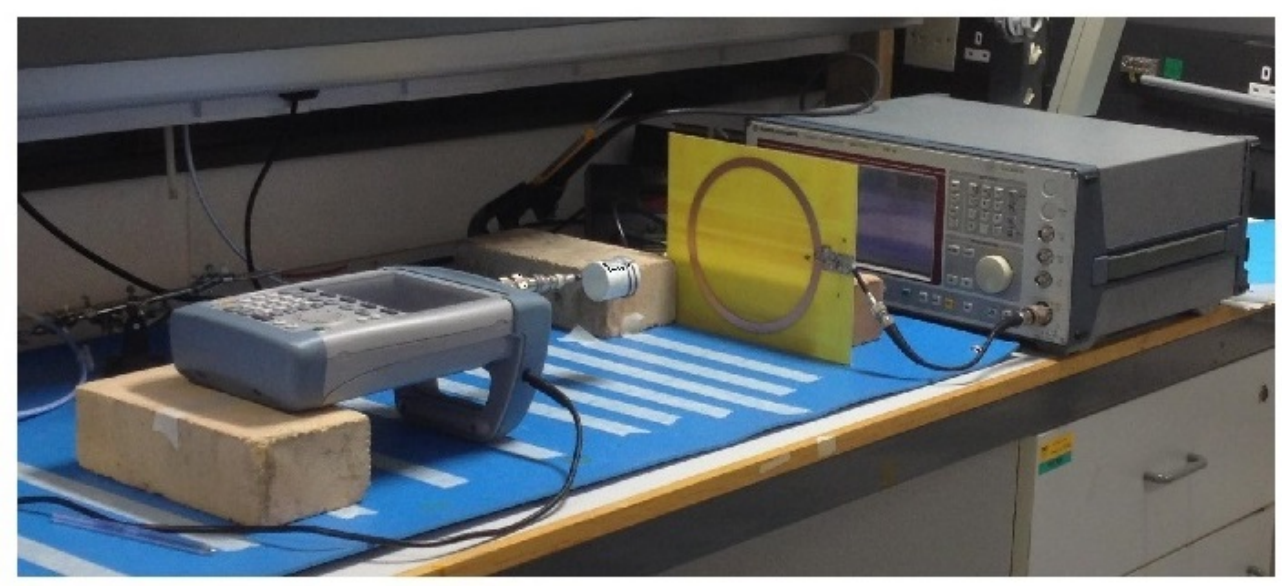

(b)

Fig. 8.

a Measured relative magnetic intensity when adjacent tags are placed in front of TTP SSRC reader antenna.

b Set up to measure the characteristic of the TTP SSRC antenna. 